\title{
Effectiveness of Mentalization based Therapy on Ego-Strength and Defense Mechanisms in People with Borderline Personality Disorder
}

\author{
Sanaz Einy*, Mohammad Narimani
}

Department of Psychology, Faculty of Educational Sciences and Psychology, University of Mohaghegh Ardabili, Ardabil, Iran

\section{A BSTRACT}

Introduction: Ego-strength and defense mechanisms are key concepts in the psychoanalytic approaches, which are important in the etiology of borderline personality disorder. Therefore, the present study was conducted with the aim of evaluating the effectiveness of mentalization based therapy (MBT) on ego-strength and defense mechanisms of people with borderline personality disorder. Materials and Methods: This was an experimental study using pretest-posttest design with a control group and follow up stage. The research sample consisted of 30 men with borderline personality disorder selected by purposive sampling and were randomly divided into experimental and control groups. MBT was performed for the experimental group. Three stages (pretest, posttest and follow up) were used to collect data from psychological inventory ego strength designed by Markstrom et al. and defense styles purposed by Andrews and colleagues. Results: The results showed that MBT was markedly effective in improving the ego strength and defense mechanisms of people with borderline personality disorder. Conclusion: MBT is a suitable strategy for improving the ego-strength and reducing neurotic and immature defense mechanisms of people with borderline personality disorder and can be used as an effective intervention method.

Key words:

1. Borderline Personality Disorder

2. Defense Mechanisms

3. Therapeutics

*Corresponding Author: Sanaz Einy

E-mail: sanaz.einy@yahoo.com 


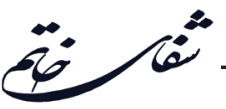

\title{
اثربخشى درمان مبتنى بر ذهنىسازى بر قدرت ايكو و مكانيسمهاى دفاعى در افراد مبتلا به اختلال شخصيت مرزى
}

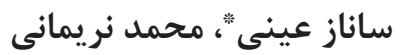

كروه روانشناسى، دانشكده علوم تربيتى و روانشناسى، دانشًاه محقق اردبيلى، اردبيل، ايران

\section{جִ}

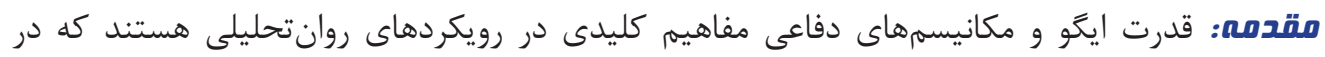

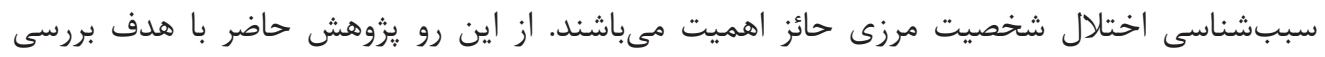

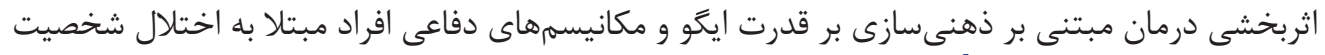

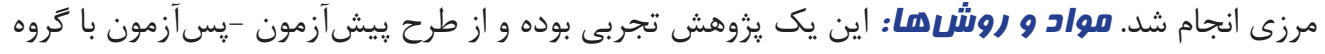

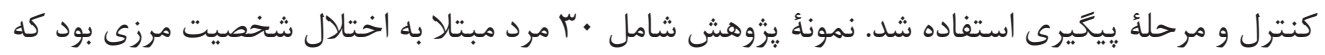

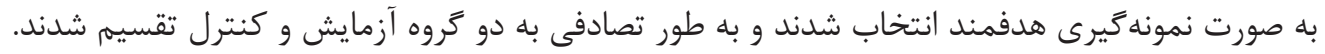

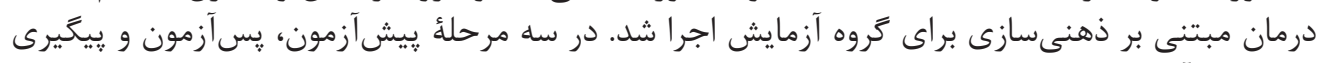

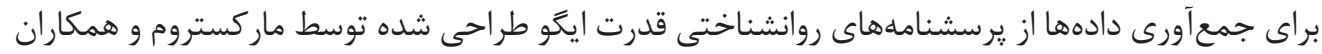

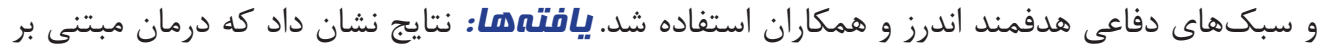

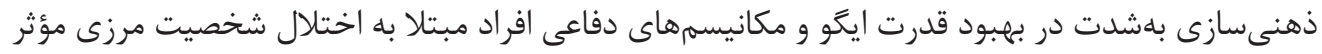

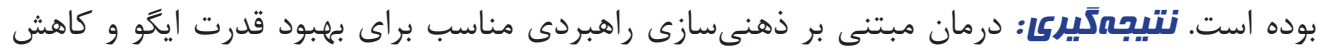

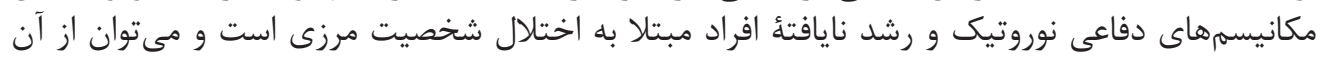
بلعنوان يك شيؤ مداخلهاى مؤثر استفاده كرد.

كليد وازمها:

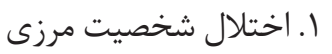

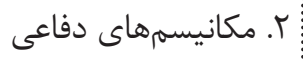

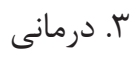




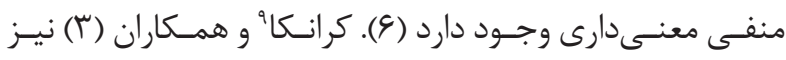

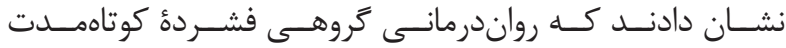

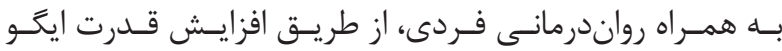

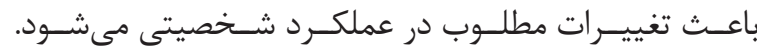

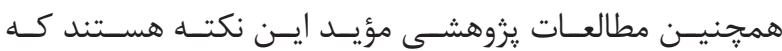

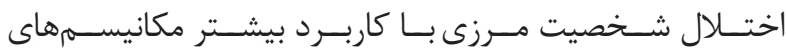

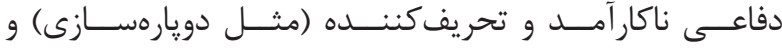

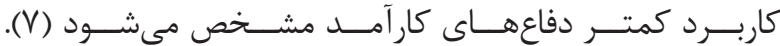

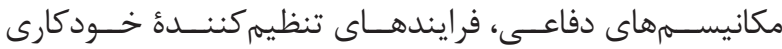

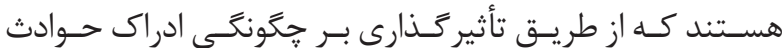

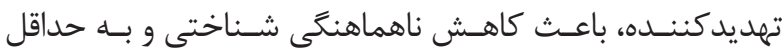

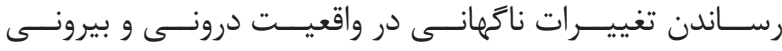

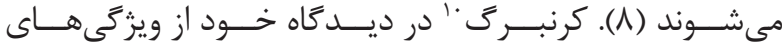

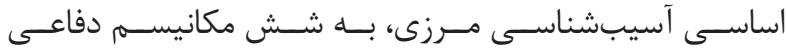

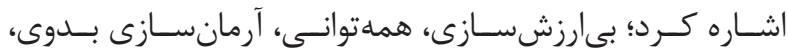

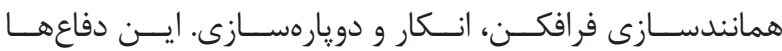

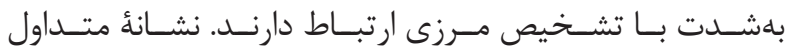

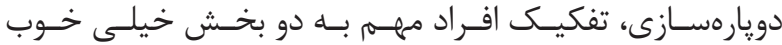

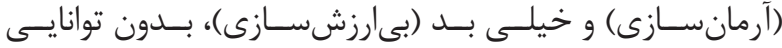

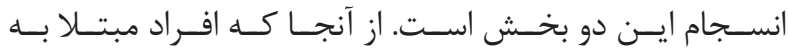

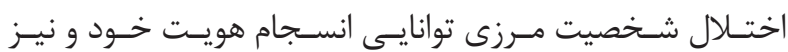

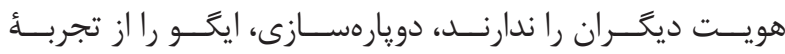

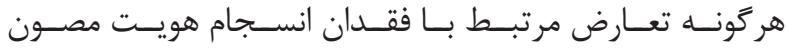

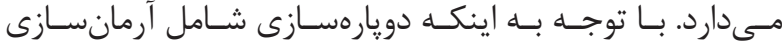

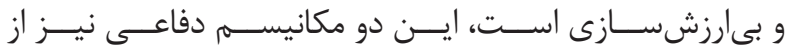

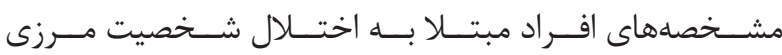

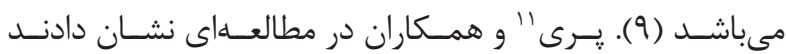

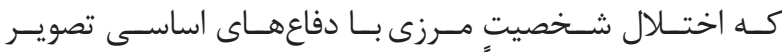

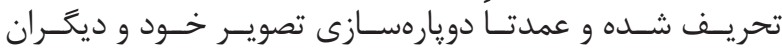

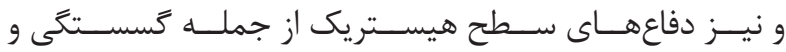

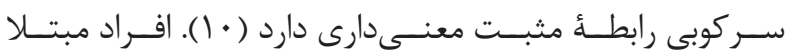

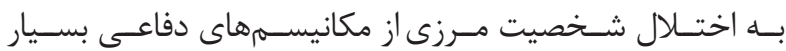

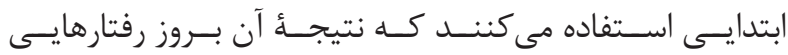

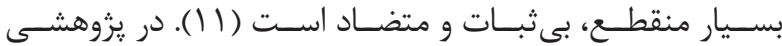

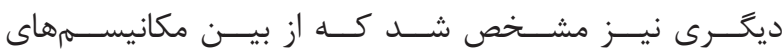

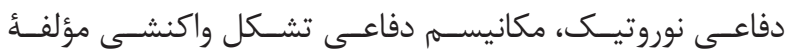

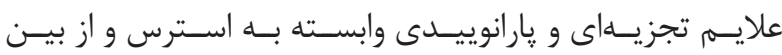

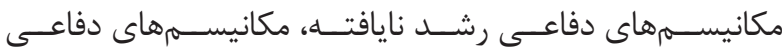

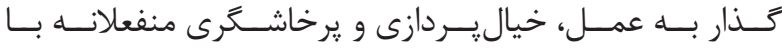

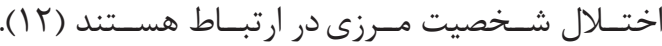

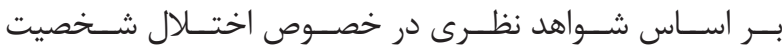

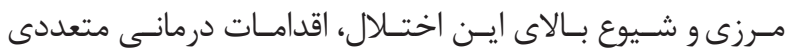

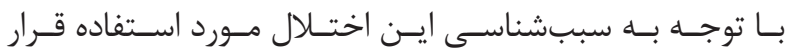

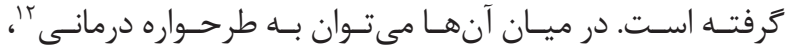

${ }^{1}$ Borderline personality disorder

${ }^{2}$ Splitting

${ }^{3}$ Projective identification

${ }^{4}$ Primary process

${ }^{5}$ Statistical manual of mental disorders, fifth edition

${ }^{6}$ Psychoanalytic

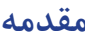

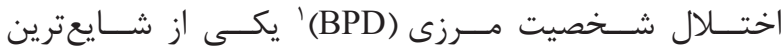

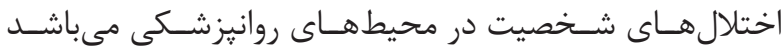

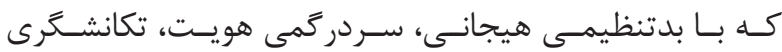

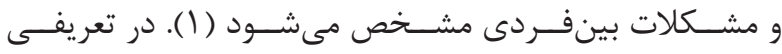

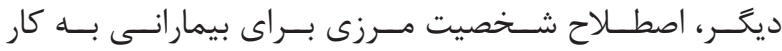

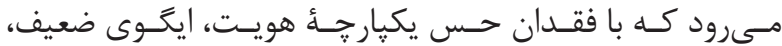

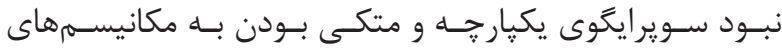

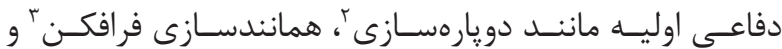

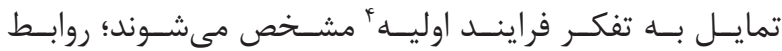

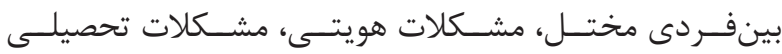

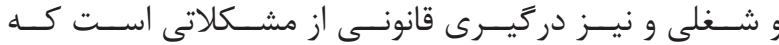

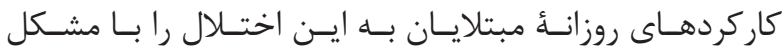

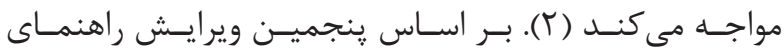

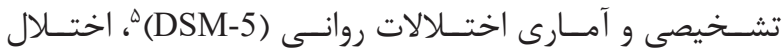

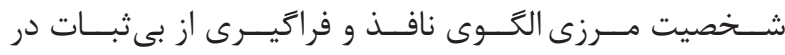

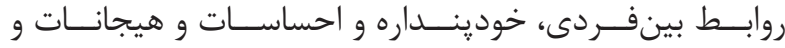

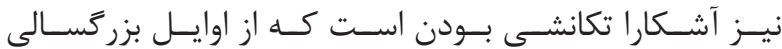

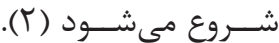

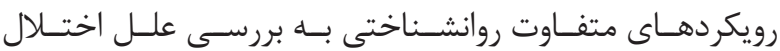

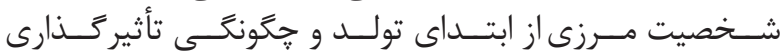

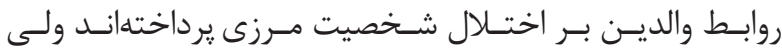

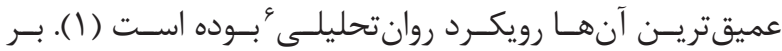

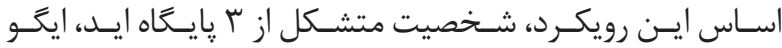

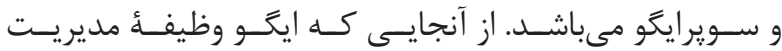

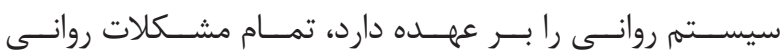

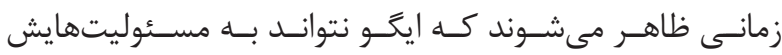

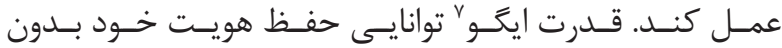

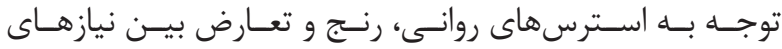

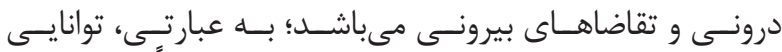

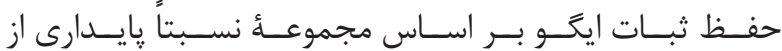

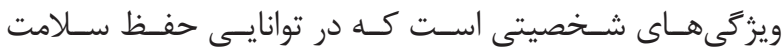

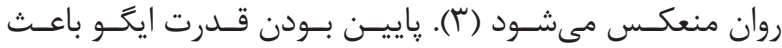

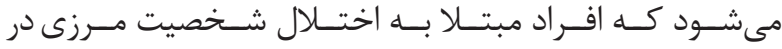

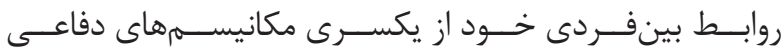

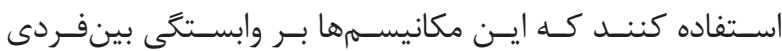

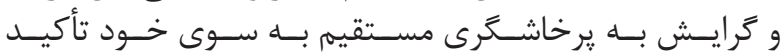

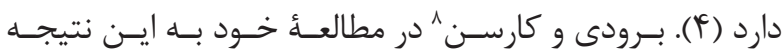

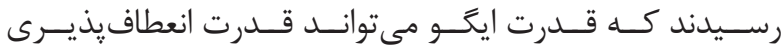

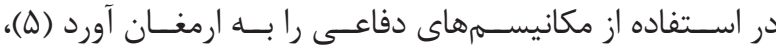

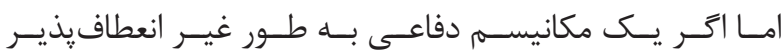

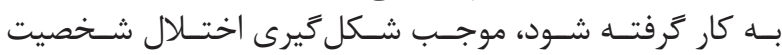

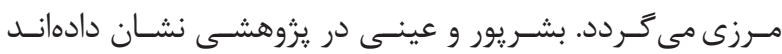

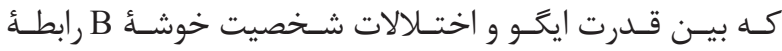

${ }^{7}$ Ego-strength

${ }^{8}$ Brody and Carson

${ }^{9}$ Cyranka

${ }^{10}$ Kernberg

${ }^{11}$ Perry

${ }^{12}$ Schema therapy 


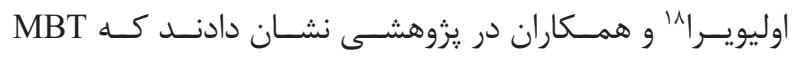

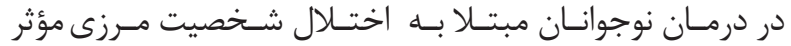

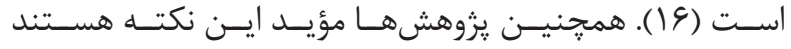

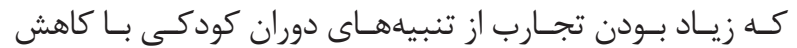

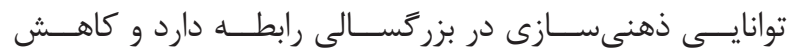

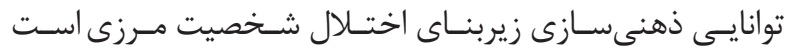

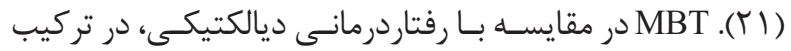

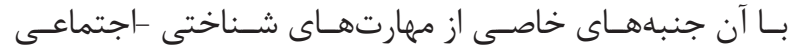

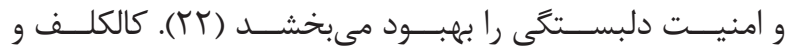

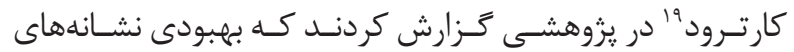

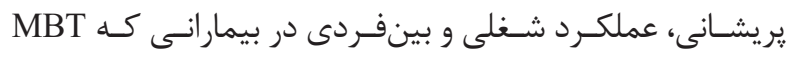

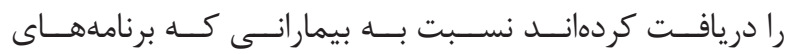

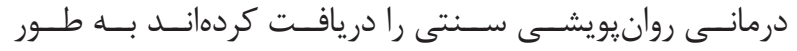

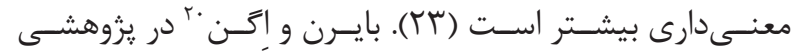

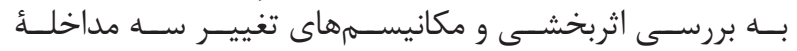

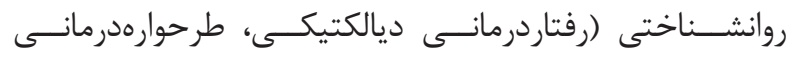

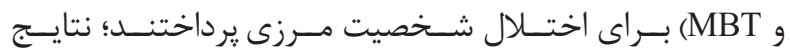

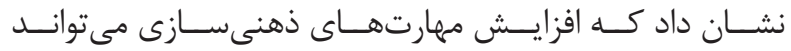

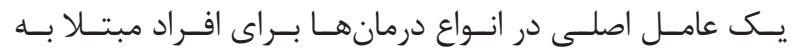

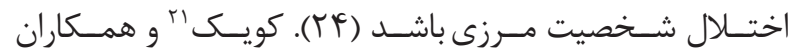

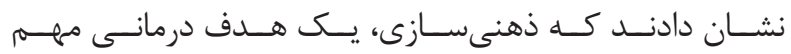

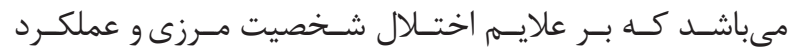

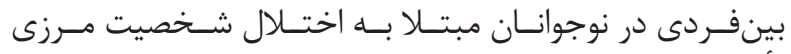

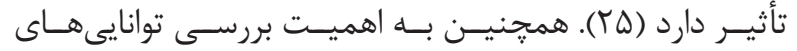

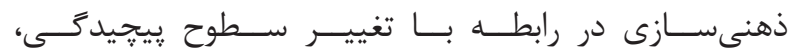

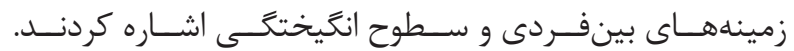

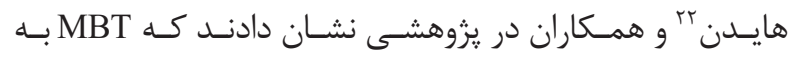

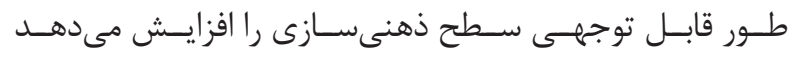

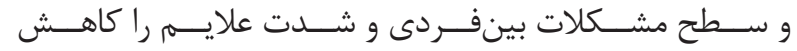

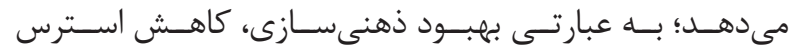

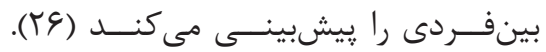

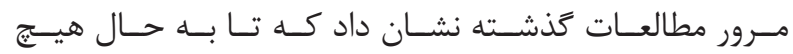

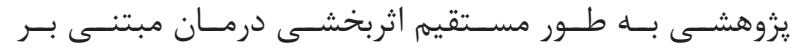

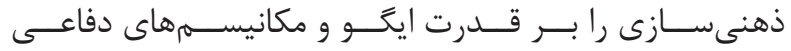

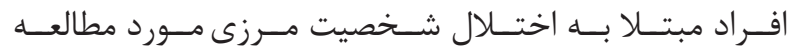

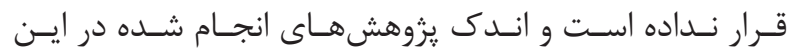

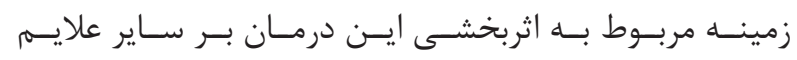

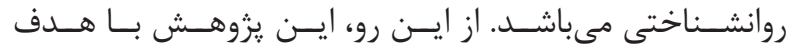

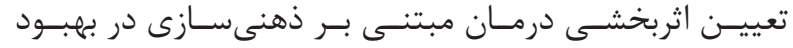

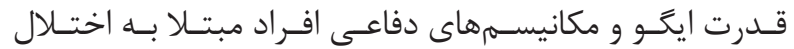

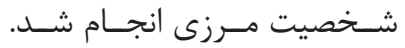

مواد و روشها

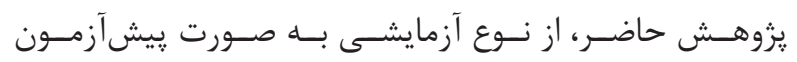

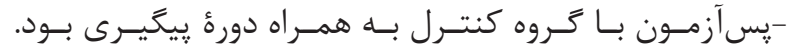

${ }^{13}$ Dialectical behavior therapy

${ }^{14}$ Transference focused psychotherapy

${ }^{15}$ Mentalization based treatment

${ }^{16}$ Bateman and Fonagy

${ }^{17}$ Brune

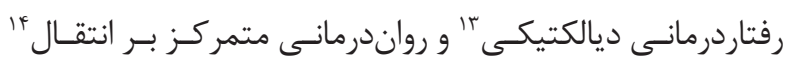

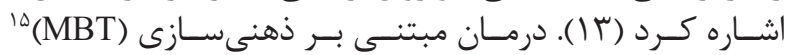

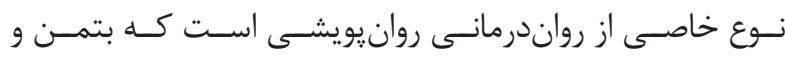

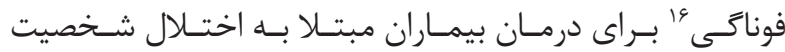

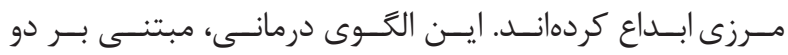

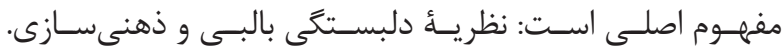

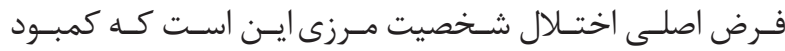

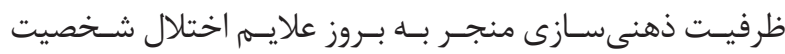

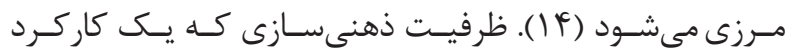

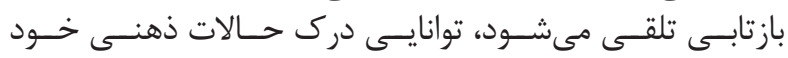

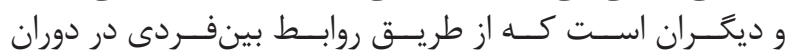

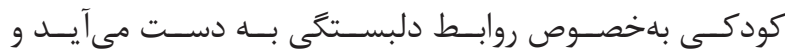

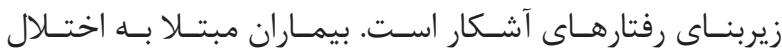

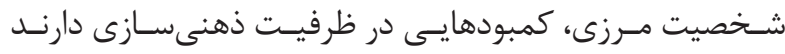

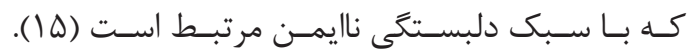

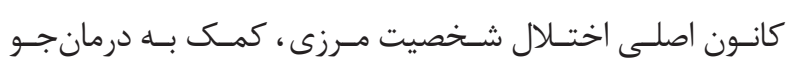

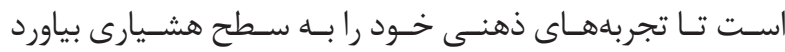

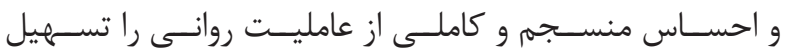

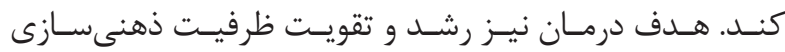

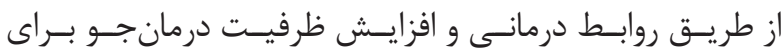

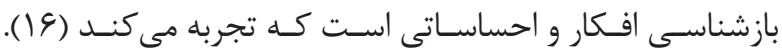

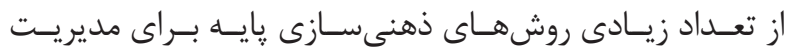

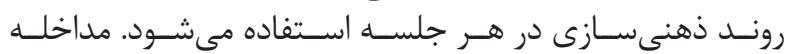

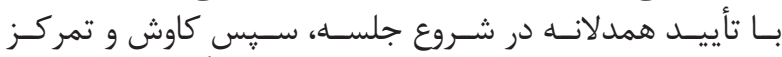

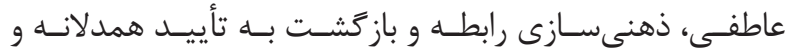

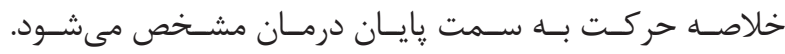

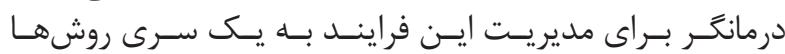

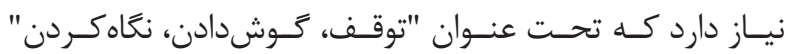

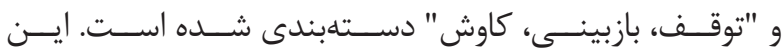

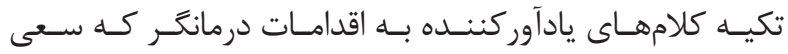

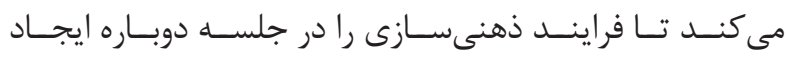

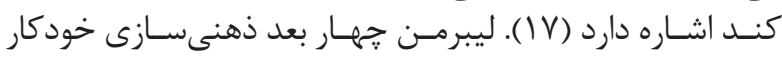

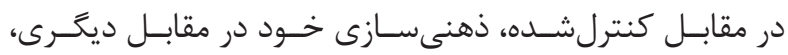

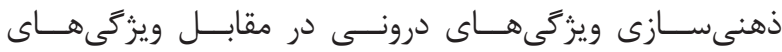

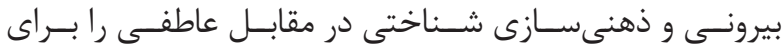

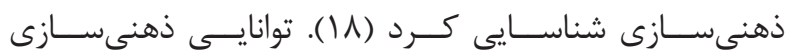

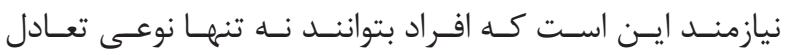

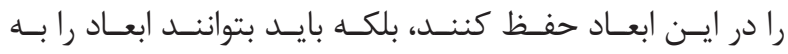

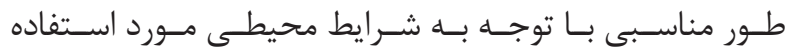

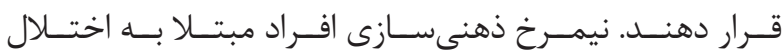

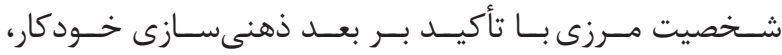

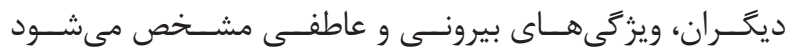

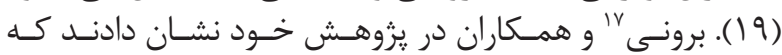

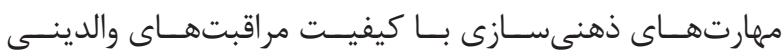

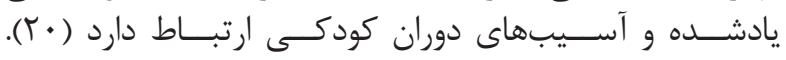

${ }^{18}$ Oliveira

${ }^{19}$ Kalleklev and Karterud

${ }^{20}$ Byrne and Egan

${ }^{21}$ Quek

${ }^{22}$ Hayden 


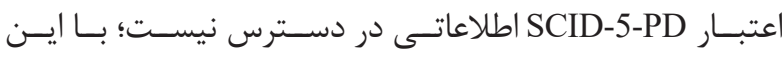

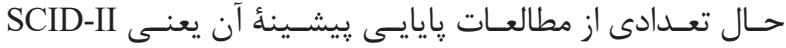

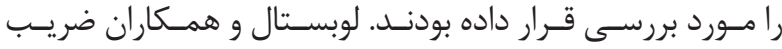

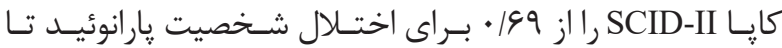

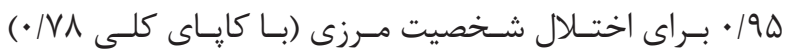

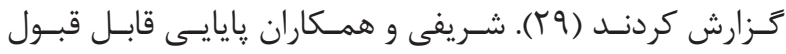

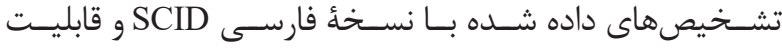

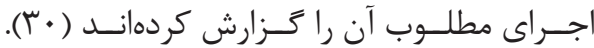

\section{III - برسشناملَ جֶند محورى ميلون}

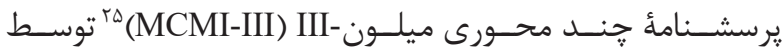

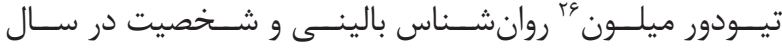

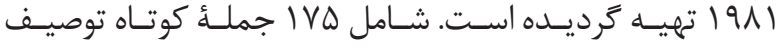

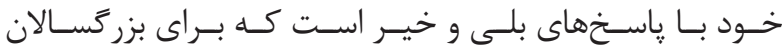

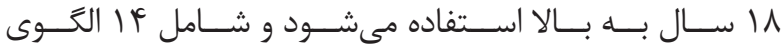

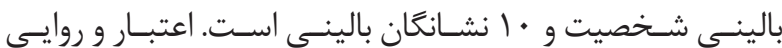

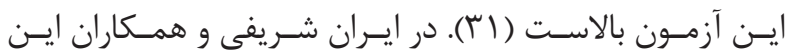

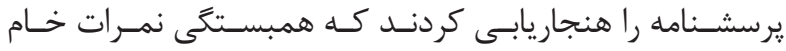

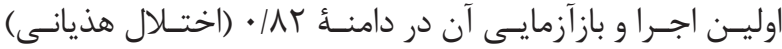

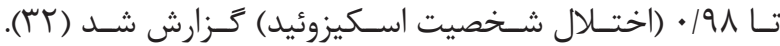

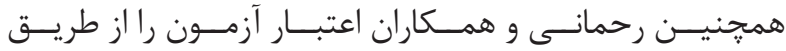

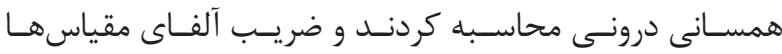

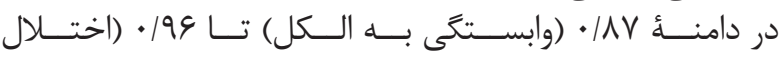

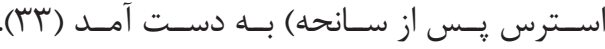

\section{r. يرسشنامة روانشناختى قدرت ايگو}

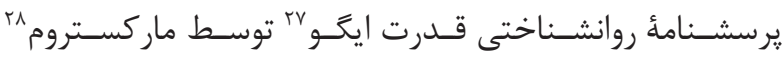

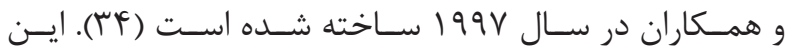

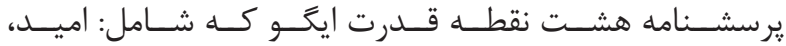

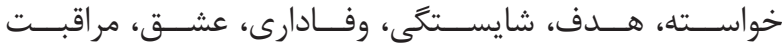

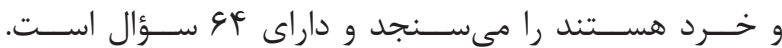

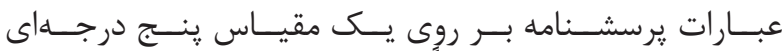

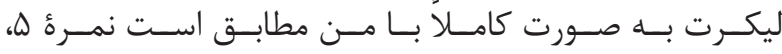

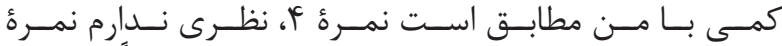

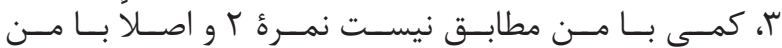

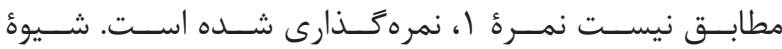

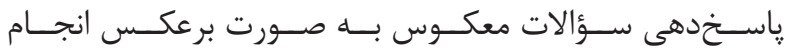

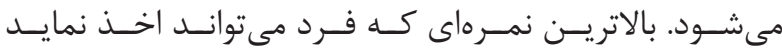

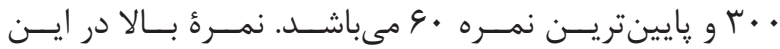

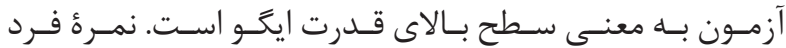

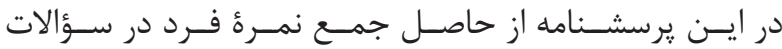

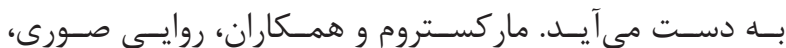

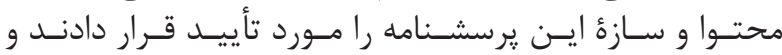

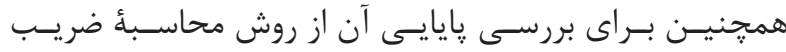

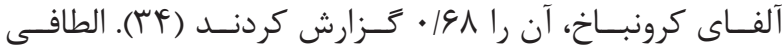

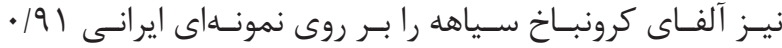

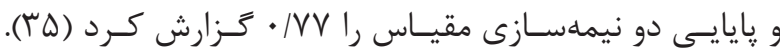

${ }^{23}$ Structured clinidal interview for DSM-5

${ }^{24}$ First

${ }^{25}$ Millon clinical multiaxial inventory- III

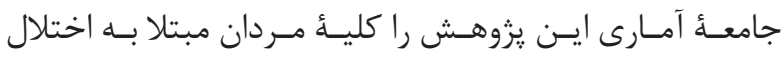

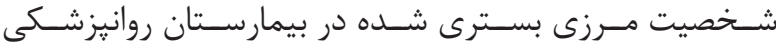

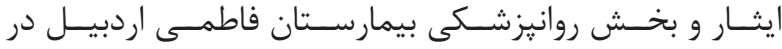

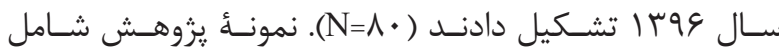

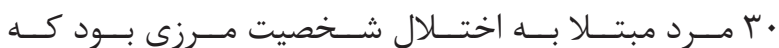

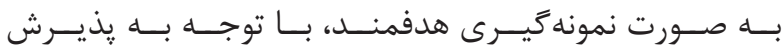

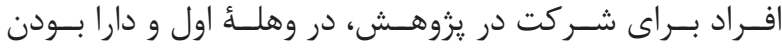

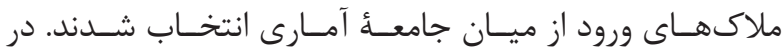

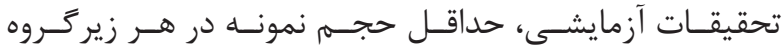

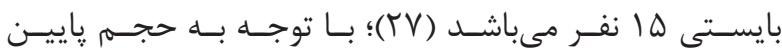

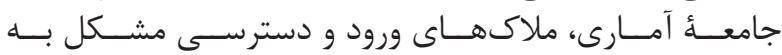

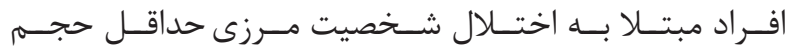

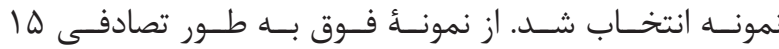

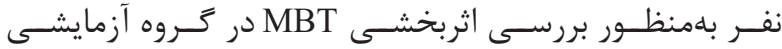

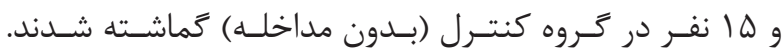

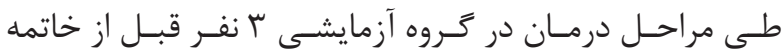

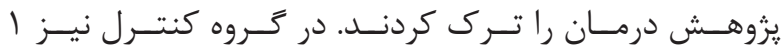

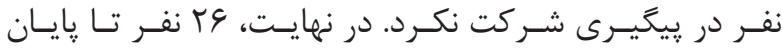

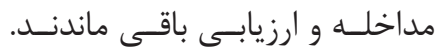

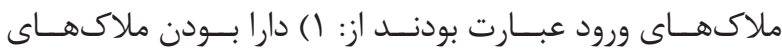

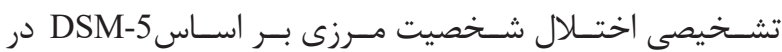

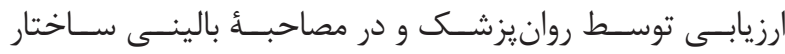

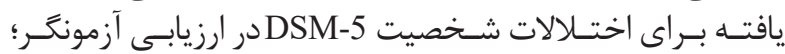

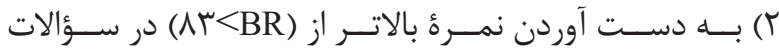

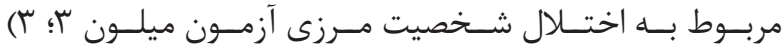

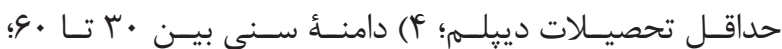

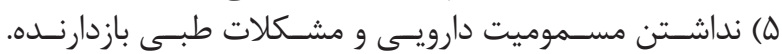

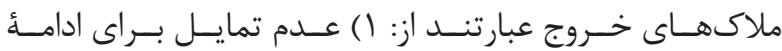

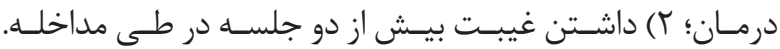

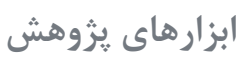

ا. مصاحبــهُ بالينــى ســـاختار يافتــهـ بـــراى اختــلالات

شــخصيت

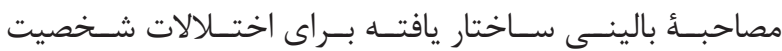
"r (SCID-5-PD) DSM-5

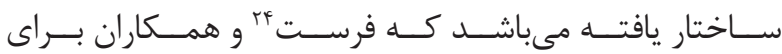

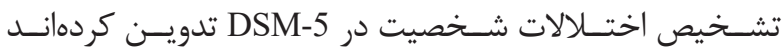

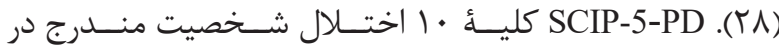

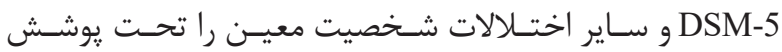

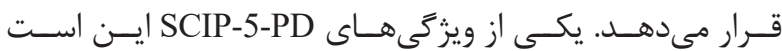

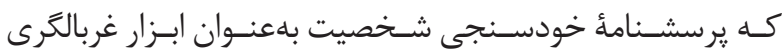

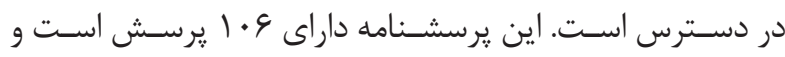

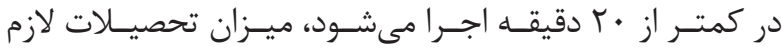

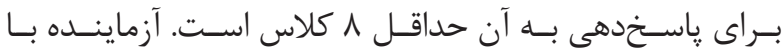

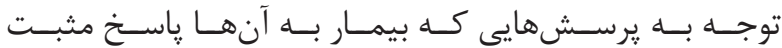

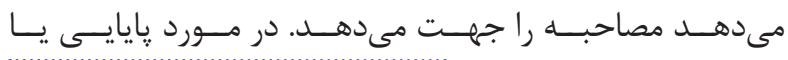

${ }^{26}$ Theodore Millon

${ }^{27}$ Psychosocial inventory of Ego strengths

${ }^{28}$ Markstrom 


\section{جلسات}

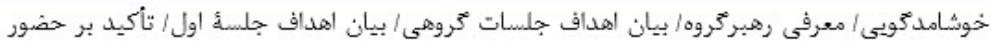

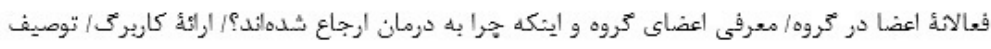

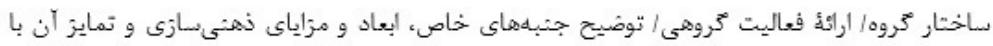

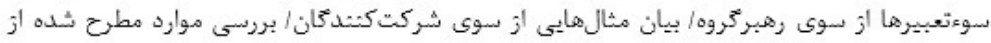

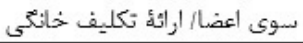

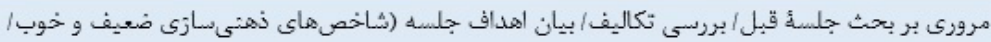

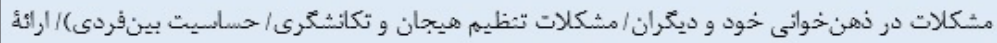

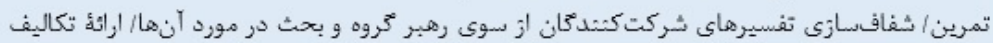

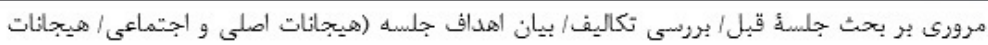

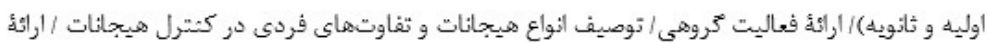
تكليف خانكي

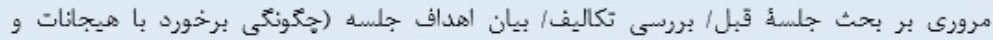

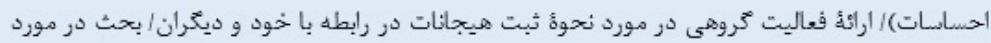

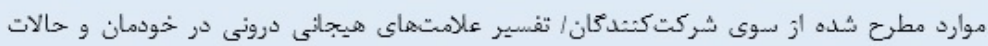

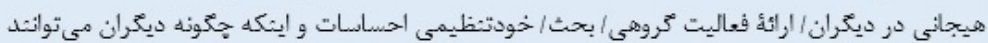

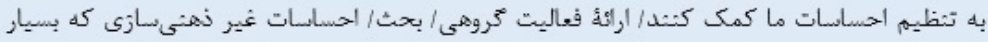

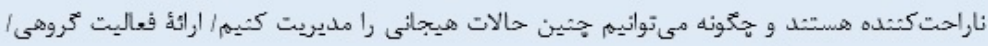

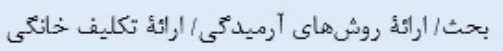

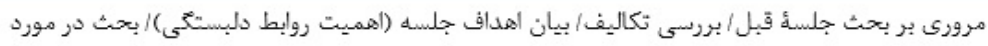

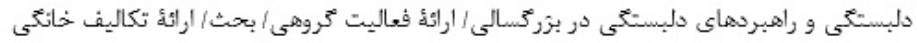

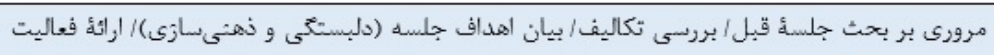

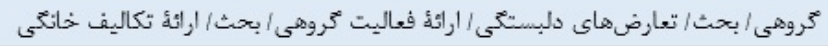

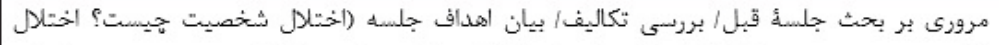

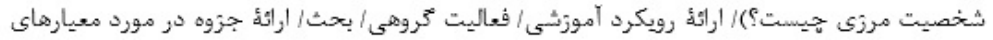
الختلال شخصيت مرزى / ارائُٔ تكاليف خائكى

مرورى بر بحث جلسـة قبل/ بروسى تكاليف/ بيان اهداف جلسه (درمان مبتثى بر ذهثى إنسازى)/ بيان

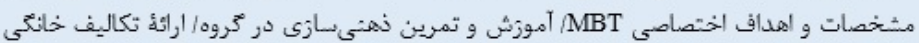

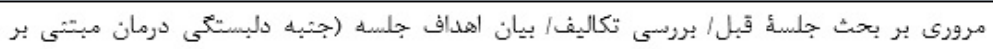

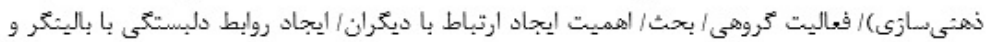

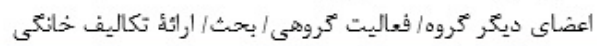

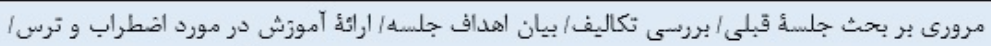

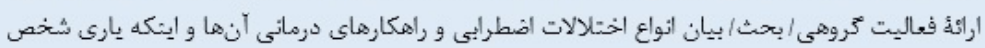

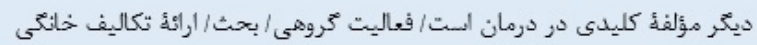

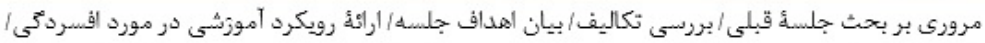

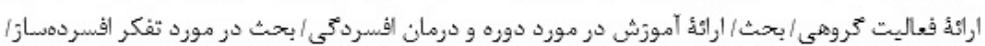

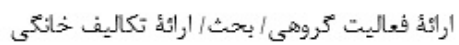
مرورى بر بحث جلسفُ قيلى/ بروسى تكاليف/ خلاصه و نتيجهد ميرى

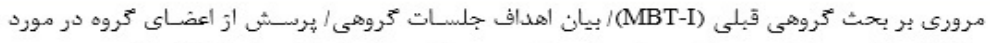

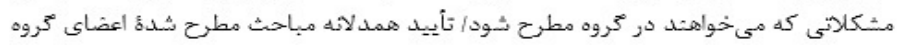

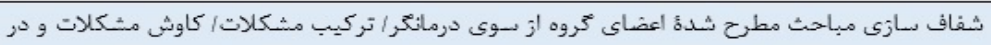

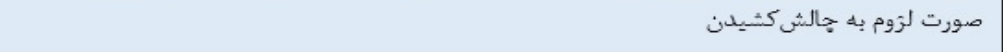
شتاسايى عاطفى و تمركز عاطفى بر روى مباحث مطرح شده از سوى اعضاى مروه آموزش در جهت ذهثى نسازى مطالب براى تسهيل اعتماد معرفتىى

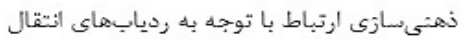

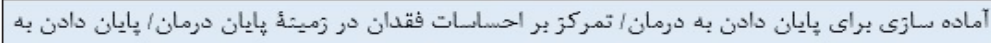
تै

اول

(ذهثى (نسازى و موضع ذهثى سازى

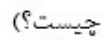

دوم

(مشكل داشتن با ذهثى دسازي بعثى ) (?d

سوم

(جرا ما هيجاناتى داريمر و هيجانات اصلى كدامتد؟؟)

مهارج (ذهثى سازى هيجانات)

MBT

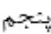

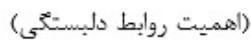

ششمر (دلبستمّى و ذهتى سازى) هفتم

BPD الختلال شخصيت حيست؟ (كيست (؟) هشتم

(درمان مبتثى بر ذهثى سازى) S

(درمان مبتثى بر ذهثى نهازى) nos (اضطراب، دلبستخى و ذهنى سازىى) يازمهم

(أفسردكى، دلبستيتى و

ذهثى مoosigo سيزنده

(حمايت و تأييد همدلانه) جهار مهمم و ياتزدهمم (شفاف سازى) شانتوهيم (شتاسايىى عاطفى و

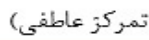
همدهم و نوزدهم (ذهثى سازى ارتباطم) 
انتخــاب و بــهـ طــور تصادفـى در دو خــروه آزمايسـش و خَــواه كمـارش شـدند.

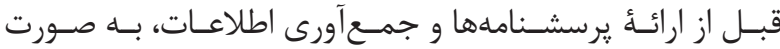

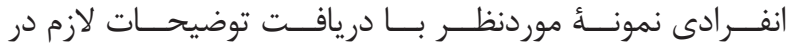

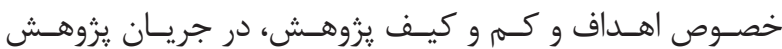

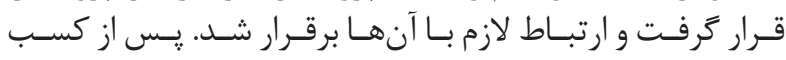

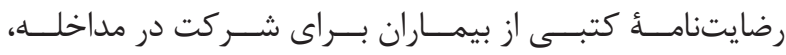

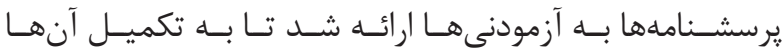

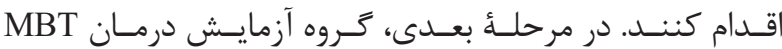

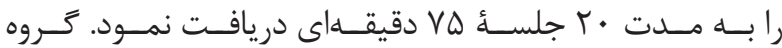

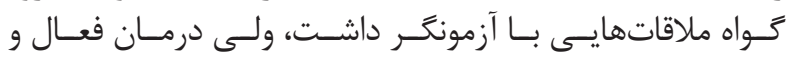

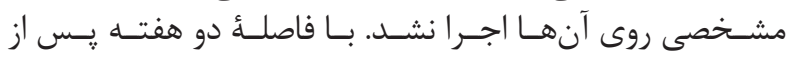

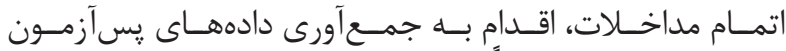

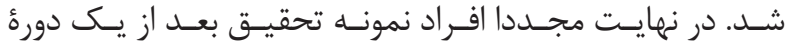

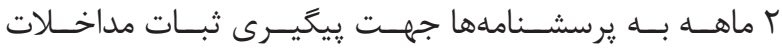

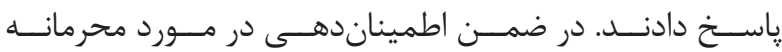

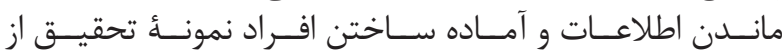

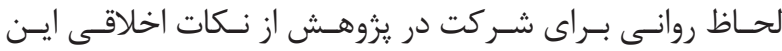

$$
\text { يزورهـش بــود. }
$$

تجزيه و تحليل دادهها

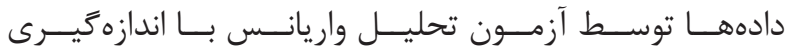

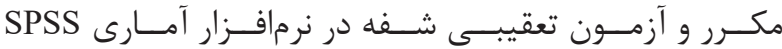

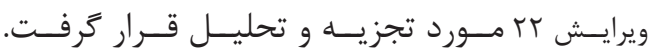

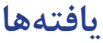

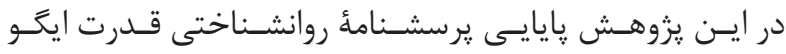

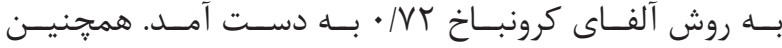

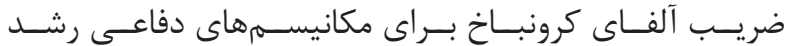

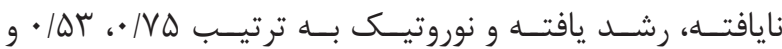

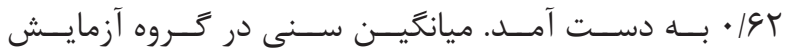
r

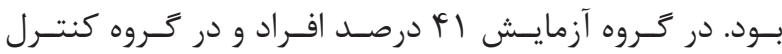

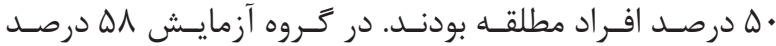

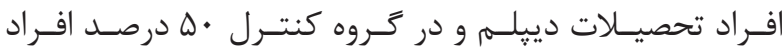

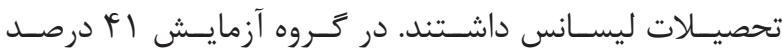

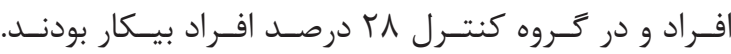

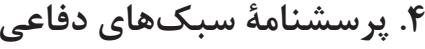

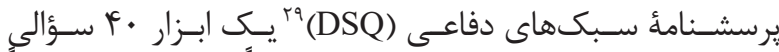

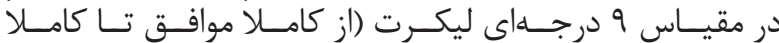

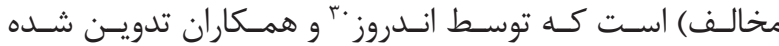

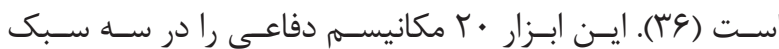

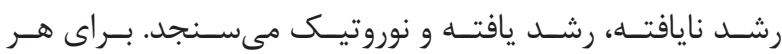

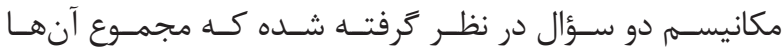

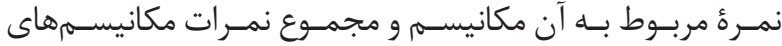

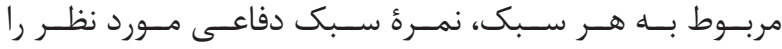

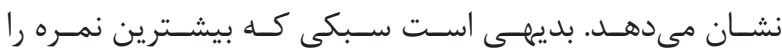

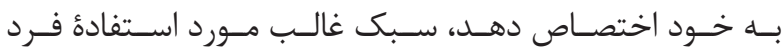

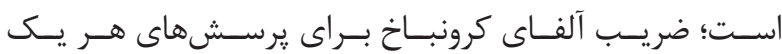

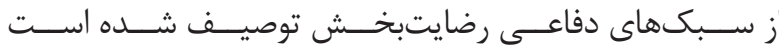

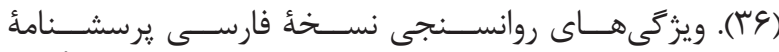

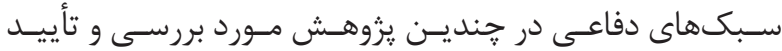
قـرار گرفتـه اسـت (rV)

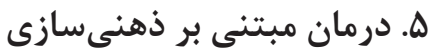

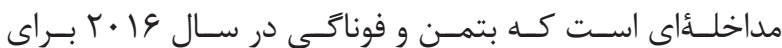

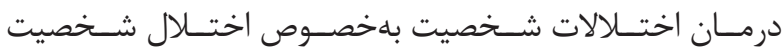

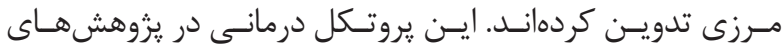

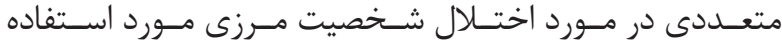

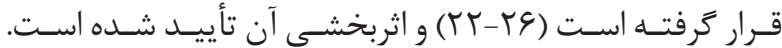

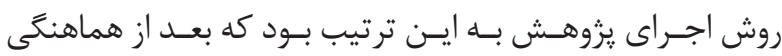

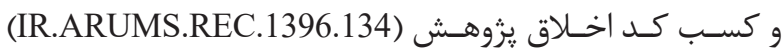

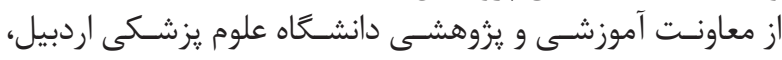

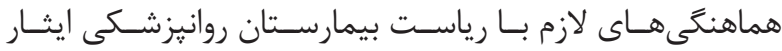

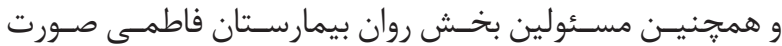

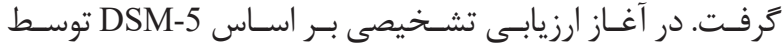

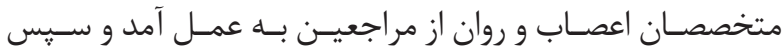

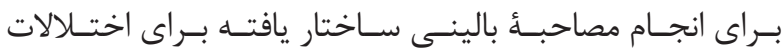

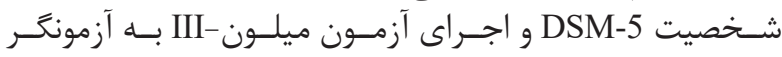

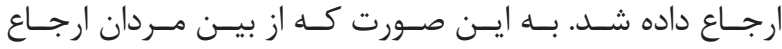

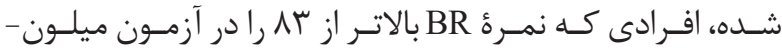
III

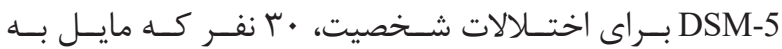

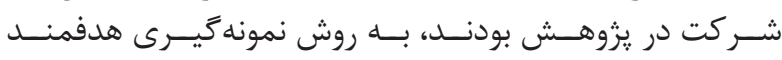

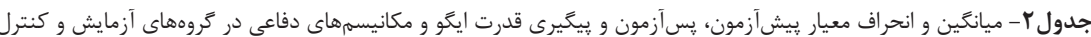

\begin{tabular}{|c|c|c|c|c|c|}
\hline هيغيرى & بس آزمون & هيش آزمون & \multirow{2}{*}{ تعداد } & \multirow{2}{*}{ تروه } & \multirow{2}{*}{ متغير } \\
\hline انحراف معيار ذميانگين & انحراف معيارثيانگين & انحراف معيار土ميانگين & & & \\
\hline$r \cdot r / q|| \varepsilon \pm / r F$ & $\mid\langle|q / 4| \pm| Q / 9 \Lambda$ & 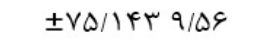 & it & آزمايش & \multirow{2}{*}{ قدرت ايخً } \\
\hline $10 V / 9 r 1 \cdot \pm / \cdot 9$ & $|\Delta r / \cdot V| 1 \pm / 9 \Delta$ & $101 / 9 \mathrm{TV} \mathrm{V} / \mathrm{TV}$ & if & كنتـرل & \\
\hline$r T / \Lambda T F \pm / V$. & $r T / \cdot \wedge r \pm / \varphi \varphi$ & $r / r r q \pm / 1$ & it & آزمايش & \multirow{2}{*}{ دفاعهاى رشد يافته } \\
\hline$\left.r T / \Delta V R \pm / T^{\prime}\right)$ & $r T / V I F \pm / T$ & $r I / r \Delta F \pm / V q$ & if & كنتـرل & \\
\hline$r) / \cdot \Lambda r \pm / V V$ & $r \cdot / V \Delta F \pm / r \Lambda$ & $r q / r \Delta \Delta \pm / / V$ & it & آزمايش & \multirow{2}{*}{ دفاعهاى نوروتيك } \\
\hline 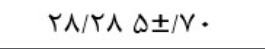 & $r \wedge / Q T Y \pm / Q I$ & 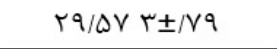 & if & كنترل & \\
\hline$f \cdot / r q \pm / q f$ & 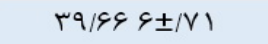 & $1.9 / 19 \mathrm{~F} \pm / \mathrm{K}$ & it & آزمايش & \multirow{2}{*}{ دفاعهاى رشد نايافته } \\
\hline$৭ \Delta / \Delta \cdot \wedge \pm / 9 \Delta$ & 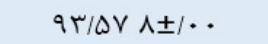 & $1 \cdot f / v \wedge \Delta \pm / f \varepsilon$ & if & كنتـرل & \\
\hline
\end{tabular}

${ }^{29}$ Defense styles questionnaire

${ }^{30}$ Andrews 


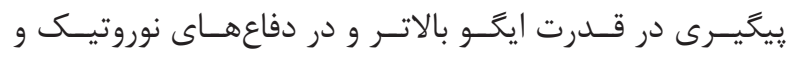

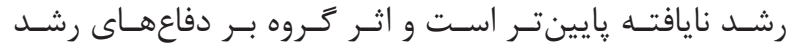

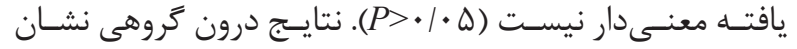

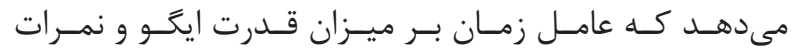

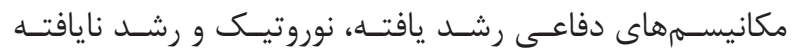

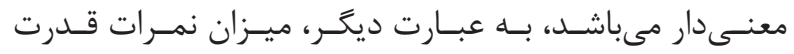

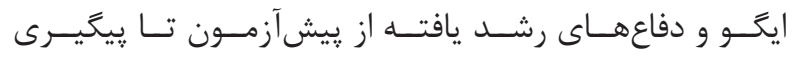

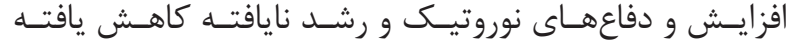

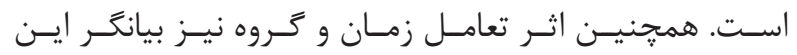

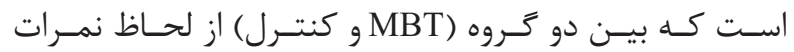

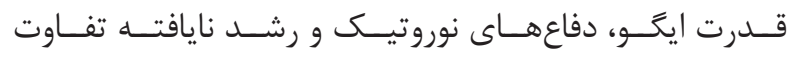

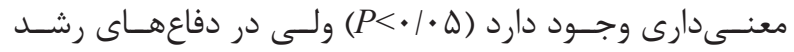

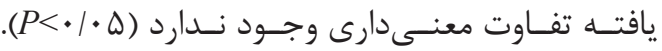

بـا هــدف مشـخص كردن ماهيـت تفــاوت متغيرهـاى وابسـتهـ

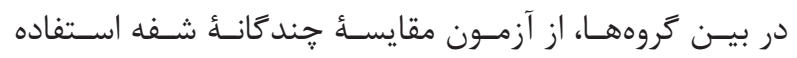

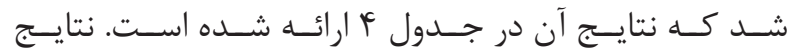

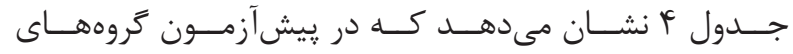

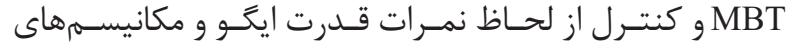

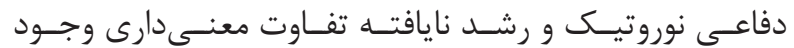

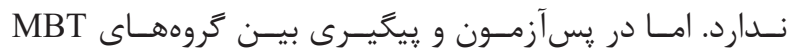

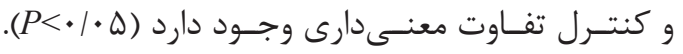

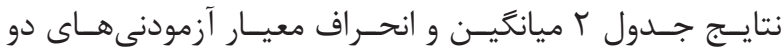

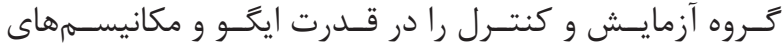

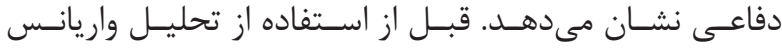

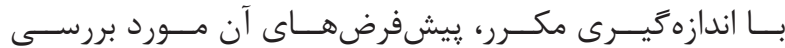

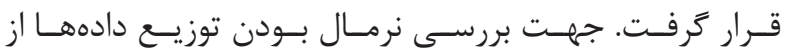

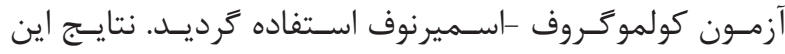

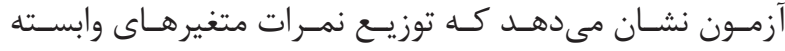

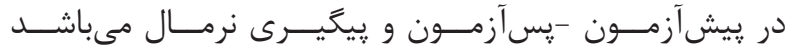

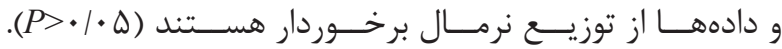

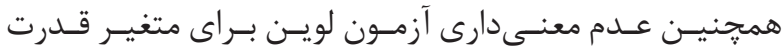

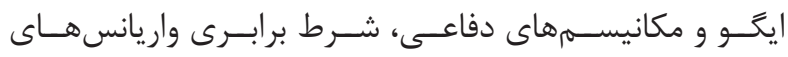

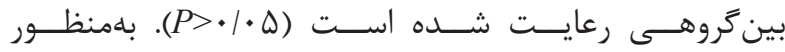

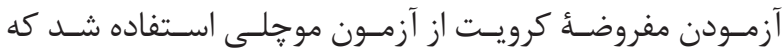

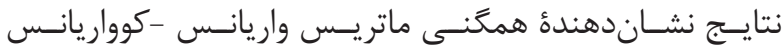

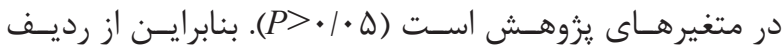

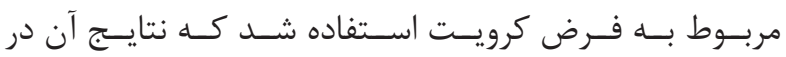

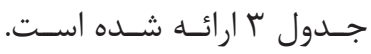

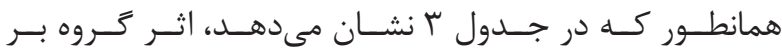

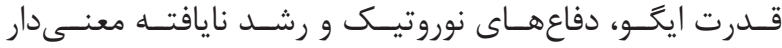

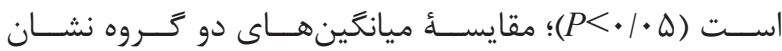

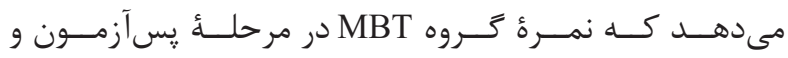

جدول بـ- نتايج تحليل واريانس با اندازمكيرى مكرر براى بررسى اثربخشى MBT و زمان بر نمرات قدرت ايكو و انواع مكانيسمهاى دفاعى.

\begin{tabular}{|c|c|c|c|c|c|c|c|c|}
\hline Partial Eta ${ }^{2}$ & $\mathbf{P}$ & $\mathbf{F}$ & MS & df & SS & \multicolumn{2}{|c|}{ منبع تغيبرات } & متثير \\
\hline$+/ V \Delta A$ & $<+1++1$ & $v Q /+\wedge r$ & Tr+rA/VY. & 1 & Tr.rA/VG. & كروه & \multirow{2}{*}{ بين ثروهى } & \multirow{5}{*}{ قدرت إيكو } \\
\hline - & - & - & rarlats & TP & $y+P p / 910$ & خطا & & \\
\hline$+/ 149+$ & $<+1++1$ & |F9/199 & $1.9 P P / 91 \mathrm{~V}$ & $r$ & rITA9/Trr & زمان & \multirow{3}{*}{ درون گروهى } & \\
\hline- & - & - & VT/PGP & iA & TPYA/TAP & خطا & & \\
\hline$+\operatorname{larA}$ & $<+1++1$ & $|r F /|+\Lambda$ & A99r/rro & $r$ & $189199 / 999$ & زمان*گكروه & & \\
\hline$+\mid++1$ & +arp & $+1 *+\gamma$ & - Irrt & 1 & . & كروه & \multirow{2}{*}{ بين ثروهى } & \multirow{5}{*}{ دكاعهاى رشد يالته } \\
\hline- & - & - & PVIATY & TP & IIFY/ATI & خطا & & \\
\hline$+/ 119$ & $+1+F A$ & r/rta & ir/lir & $r$ & TS/TTA & زمان & \multirow{3}{*}{ درون گروهى } & \\
\hline- & - & - & $91+91$ & is & 199/9T9 & خطا & & \\
\hline$+1+19$ & $+/ V T$ & $+|r r|$ & lirer & $r$ & $\mathrm{r} / \mathrm{GAV}$ & زمان*گكروه & & \\
\hline$+/ r+9$ & $+\mid++r$ & 1./YQP & $\Delta+T / F 99$ & 1 & $\Delta+T / P 49$ & كروه & \multirow{3}{*}{ بين ثروهى } & \multirow{5}{*}{ ناعهاى نوروتيك } \\
\hline- & - & - & PG/VTP & TP & $\| r \mid / r v y$ & خطا & & \\
\hline$+10+1$ & $+\mid++1$ & TF/VG. & $199 / 199$ & $r$ & rıq/vq. & زمان & & \\
\hline- & - & - & $V|A v|$ & is & rVY/ATo & خطا & \multirow{2}{*}{ درون ثروهى } & \\
\hline$+/ T r+$ & $+\mid+1$ & $\mid 9 / .99$ & $11+/ V F 1$ & $r$ & TTI/PAT & زمان*گكروه & & \\
\hline$+/ 9 T 1$ & $<+1++1$ & Trq/AIr & TFQYAlG9A & 1 & TFqYA/99A & كروه & \multirow{2}{*}{ بين ثروهى } & \multirow{5}{*}{ دكاع هاى رشد ثايالته } \\
\hline- & - & - & $19 / 799$ & TP & TIFT/PG. & خطا & & \\
\hline -/9९q & $<+\mid++1$ & $P P Y|q P|$ & $\operatorname{IT\Delta \Lambda N/T\Lambda +}$ & $r$ & TOIVG/DQQ & زمان & \multirow{3}{*}{ درون كروهى } & \\
\hline - & - & - & TN/ITI & in & ITFQ/ATS & خطا & & \\
\hline$+/ 9+9$ & $<+1++1$ & rra/DGV & grre/aps & $r$ & irevrinar & زمان* كروه & & \\
\hline
\end{tabular}




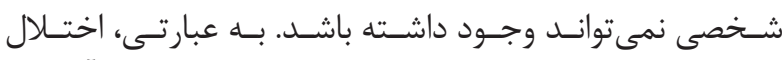

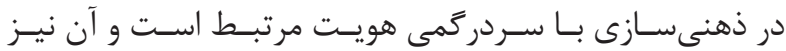

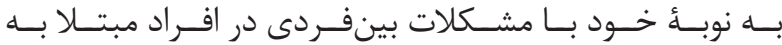

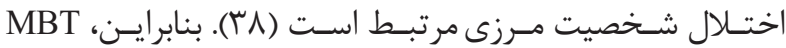

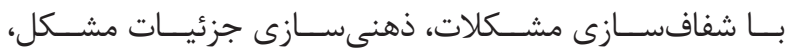

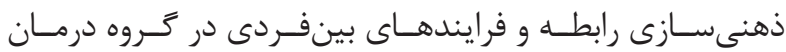

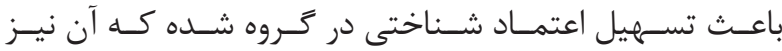

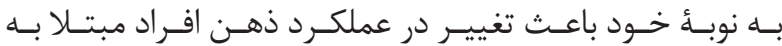

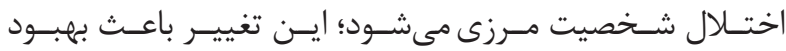

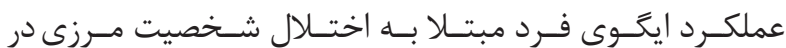

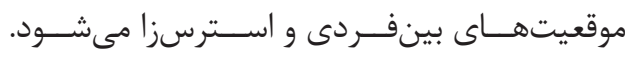

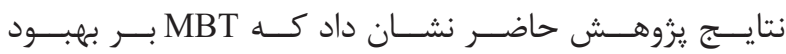

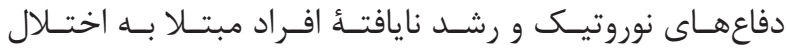

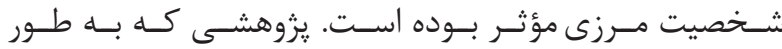

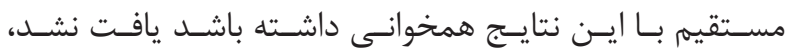

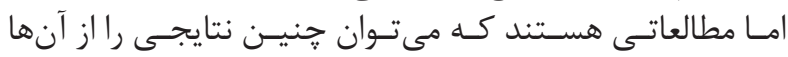

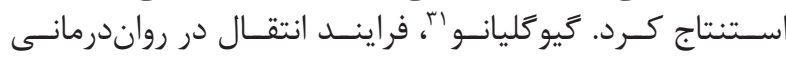

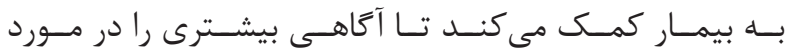

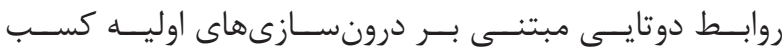

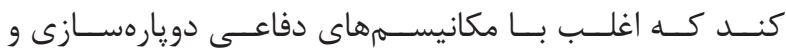

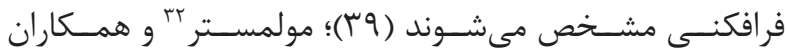

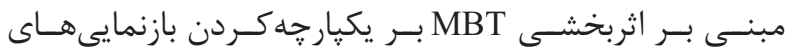

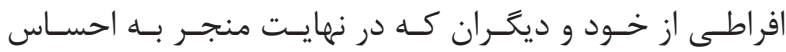

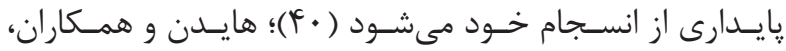

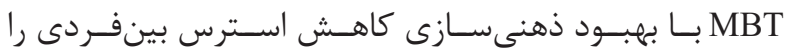

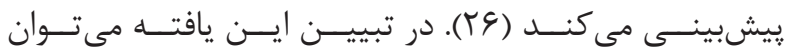

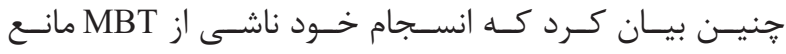

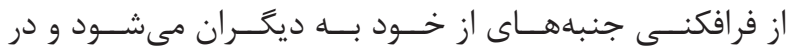

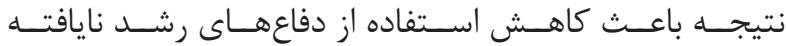

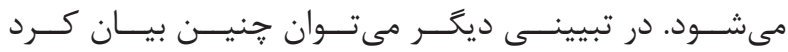

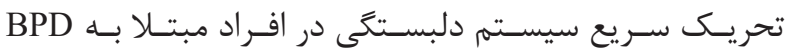

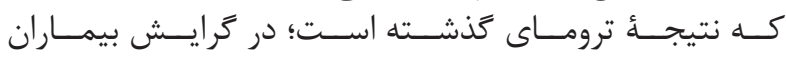

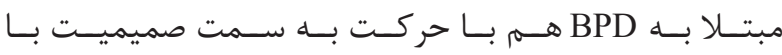

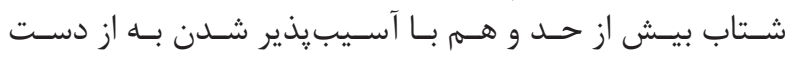

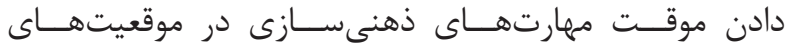

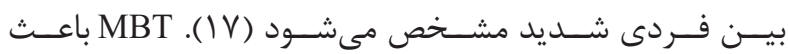

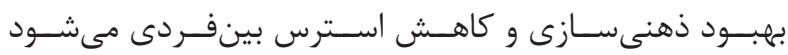

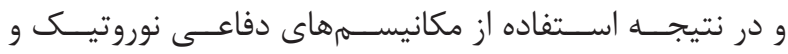

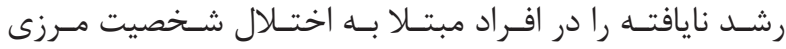

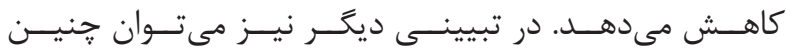

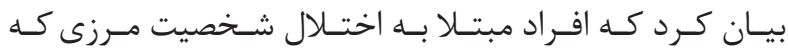

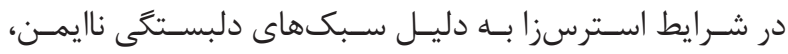

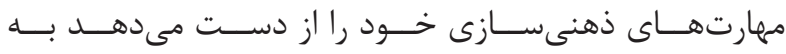

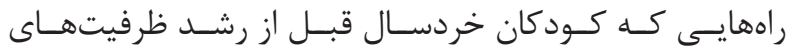

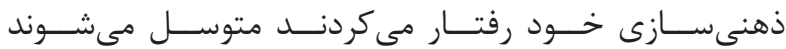

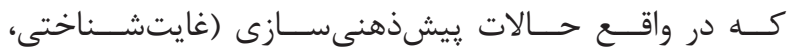

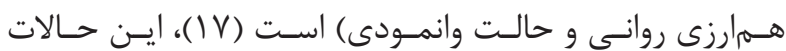

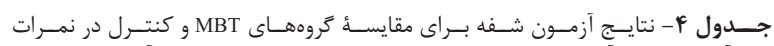

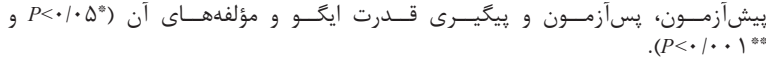

\begin{tabular}{|c|c|c|}
\hline مقايسهُ ميانكين دو كروه & دوره & متغير \\
\hline$-\Lambda / I V$ & بيش آزمون & \multirow{3}{*}{ قدرت ايكو } \\
\hline Sr/rF** & يس آزمون & \\
\hline$F \Delta / 9 \Lambda^{* * *}$ & ييعيرى & \\
\hline.$- / T T$ & ييش آزمون & \multirow{3}{*}{ دفاعهاى نوروتيك } \\
\hline$-\Lambda / 1 \mathbf{V}^{* * *}$ & يس آزمون & \\
\hline$-V / T+*$ & ييكيرى & \\
\hline $1 / \mathrm{rA}$ & ييش آزمون & \multirow{3}{*}{ دفاع هاى رشد نايافته } \\
\hline$-\Delta r / 9 \cdot * *$ & هس آزمون & \\
\hline$-\Delta \Delta / 1 \varphi^{* * *}$ & بيخيرى & \\
\hline
\end{tabular}

بحث و نتيجه كيرى

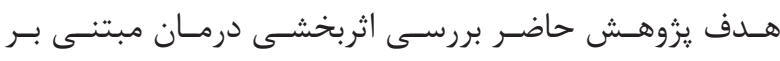

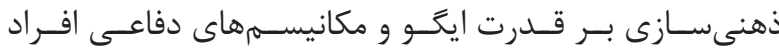

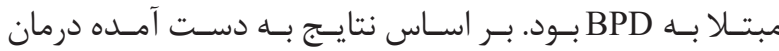

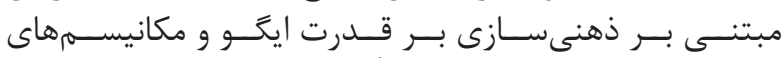

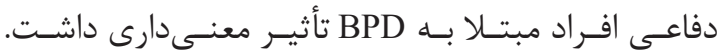

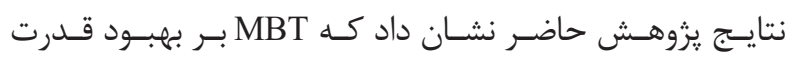

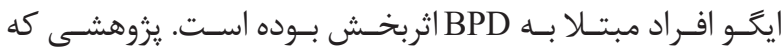

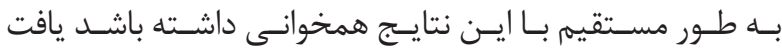

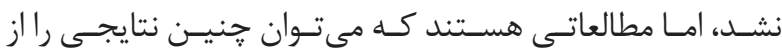

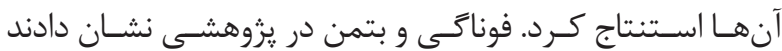

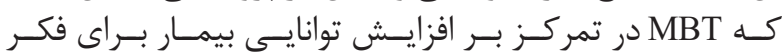

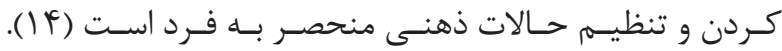

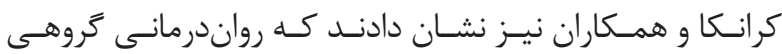

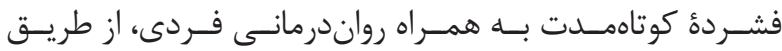

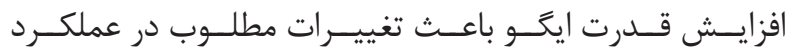

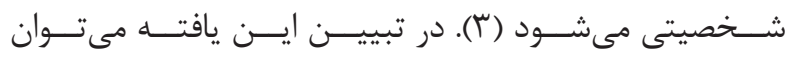

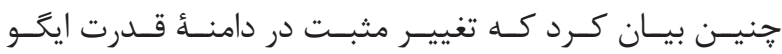

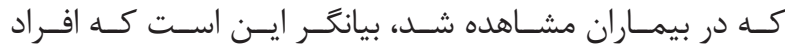

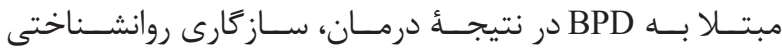

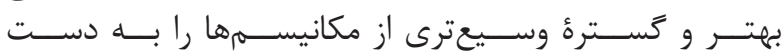

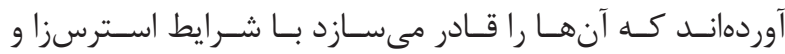

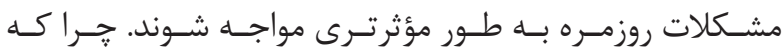

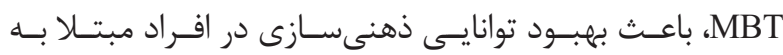

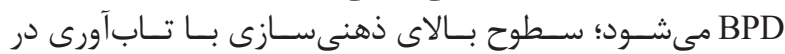

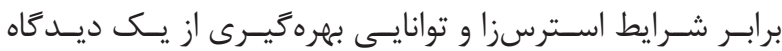

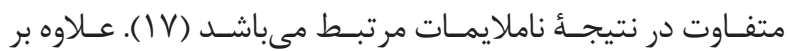

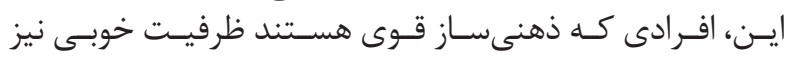

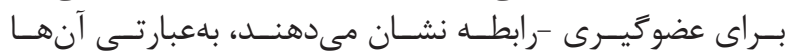

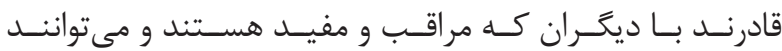

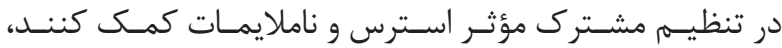

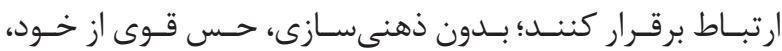

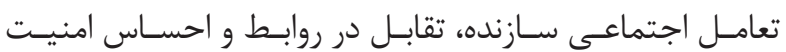

${ }^{31}$ Giugliano

${ }^{32}$ Meulemeester 


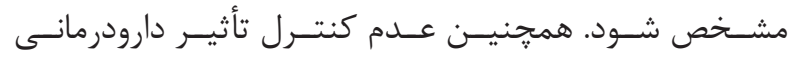

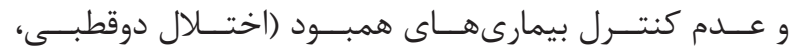

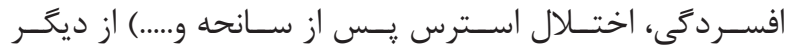

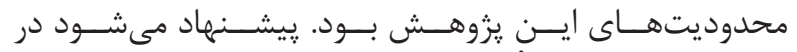

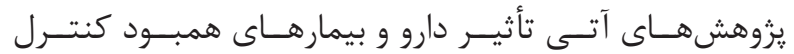

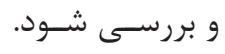

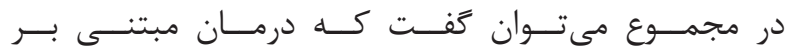

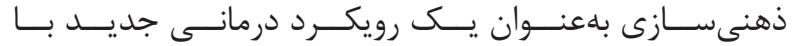

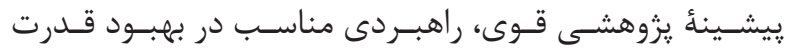

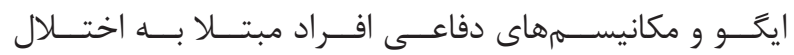

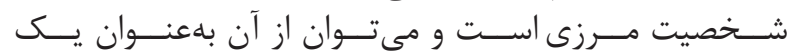

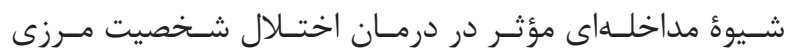
اسـتفاده كـرد. تشكر و قدردانى

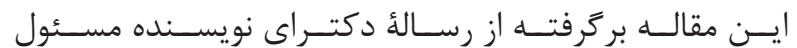

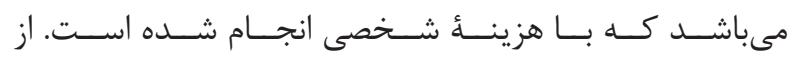

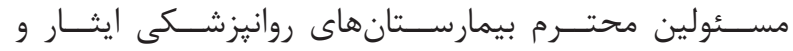

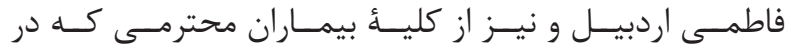

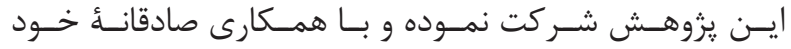

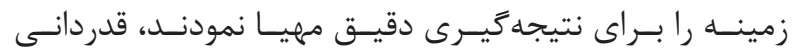
مى شـــود.

1. American Psychiatric Association. Diagnostic and $\mathrm{s}$ tatistical manual of mental disorders DSM-5. $5^{\text {th }}$ ed. Arlington (VA): American Psychiatric Association Publishing; 2013.

2. Sadock BJ, Sadock VA, Ruiz P. Kaplan and sadock's synopsis of psychiatry: Behavioral sciences/clinical psychiatry. $11^{\text {th }}$ ed. Wolters Kluwer: Lippincott Williams \& Wilkins; 2014.

3. Cyranka K, Rutkowski K, Mielimaka M, Sobanski JA, Klasa K, Müldner-Nieckowski L, et al. Changes in ego strength in patients with neurotic and personality disorders treated with a short-term comprehensive psychodynamic psychotherapy. Psychiatr Pol. 2018; 52(1): 115-27.

4. Presniak MD, Olson TR, Macgregor MW. The role of defense mechanisms in borderline and antisocial personalities. Journal of Personality Assessment. 2010; 92(2): 137-45.

5. Brody S, Carson CM. Brief report: self-harm is associated immature defense mechanisms but not substance use in a nonclinical Scottish adolescent sample. Journal of Adolescence. 2014; 35(3): 765-7.

6. Basharpoor S, Einy S. Predicting personality disorders based on Zuckerman's alternative five -factor model
بـا بـهـ كارگيــرى دفاعهــاى رشـد نايافتـهـه و نوروتيـك باعـث

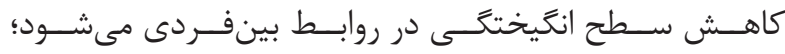

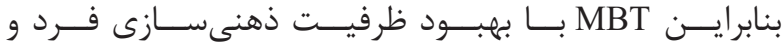

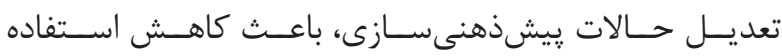

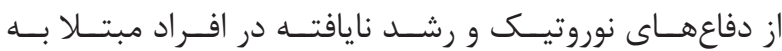

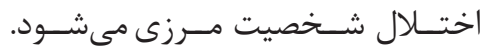

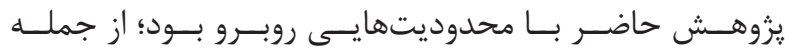

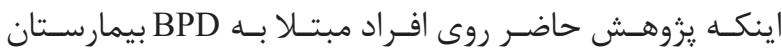

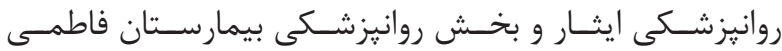

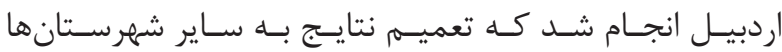

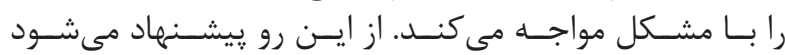

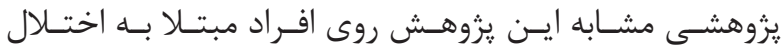

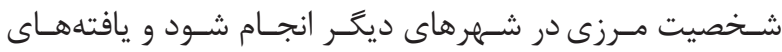

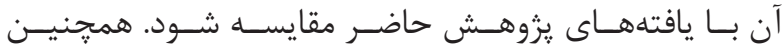

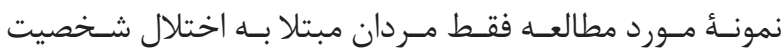

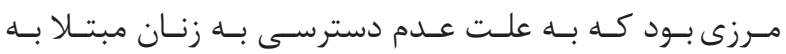

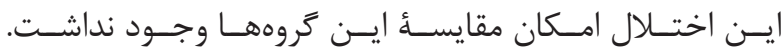

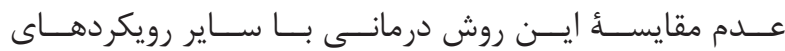

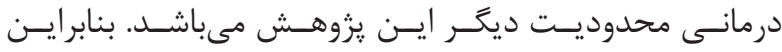

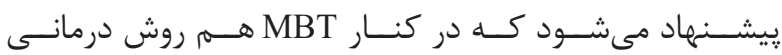

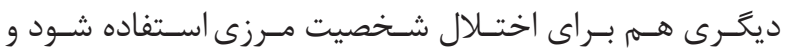

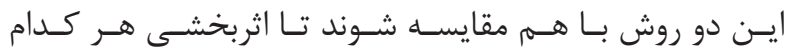

منابع

and ego strength. Journal of Shahrekord University Medical Sciences. 2017; 19(5): 84-93.

7. Bond M. Empirical studies of defense style: relationships with psychopathology and change. Harv Rev Psychiatry. 2004; 12(5): 263-78.

8. Vaillant GE. Triumphs of experience: the men of the harvard grant study. Cambridge, MA: Belknap of Harvard UP. 2012.

9. Presniak MD. Can defense mechanisms aid in our differentiation of borderline and antisocial personalities? PhD thesis. University of Saskatchewan, SK. 2008.

10. Perry JC, Presniak MD, Olson TR. Defense mechanisms in schizotypal, borderline, antisocial, and narcissistic personality disorders. Psychiatry. 2013; 76(1): 32-52.

11. Sinha BK, Watson DC. Personality disorder clusters and defense style questionnaire. Psychology and Psychotherapy: Theory, Research and Practice. 2004; 77(1): 55-66.

12. Nohi S, Hasani J. The relationship between cognitive emotion regulation strategies and defense mechanisms with borderline personality disorder. Journal of Thought and Behavior in Clinical Psychology. 2017; 
11(43): 57-66.

13. Dimeff LA, Koerner K. Dialectical behavior therapy in clinical practice. New York: Guillford press; 2007.

14. Bateman A, Fonagy P. Mentalization based treatment for borderline personality disorder. World Psychiatry. 2010; 9(1): 11-5.

15. Bateman A, Fonagy P. Impact of clinical severity on outcomes of mentalization- based treatment for borderline personality disorder. Br J Psychiatry. 2013; 203(3): 221-7.

16. Oliveira CD, Rahioui H, Smadja M, Gorsane MA, Louppe F. Mentalization based treatment and borderline personality disorder. Encephale. 2017; 43(4): 340-5.

17. Bateman A, Fonagy P. Mentalization-based treatment for personality disorders. $1^{\text {th }}$ ed. United Kingdom: Oxford University Press; 2016.

18. Lieberman MD. Social cognitive neuroscience: A review of core processes. Annu Rev Psychol. 2007; 58: 259-89.

19. Sharp C. The social-cognitive basis of BPD: A theory of hypermentalizing. Sharp C, Tackett JL. Handbook of borderline personality disorder in children and adolescents. New York, NY: Springer; 2014.

20. Brune M, Walden S, Edel MA, Dimaggio G. Mentalization of complex emotions in borderline personality disorder: The impact of parenting and exposure to trauma on the performance in a novel cartoon-based task. Comprehensive Psychiatry. 2016; 64: $29-37$

21. Petersen R, Brakoulias V, Langdon R. An experimental investigation of mentalization ability in borderline personality disorder. Compr Psychiatry. 2016; 64: 12-21.

22. Edel MA, Raaff V, Dimaggio G, Buchheim A, Brune M. Exploring the effectiveness of combined mentalization- based group therapy and dialectical behaviour therapy for inpatients with borderline personality disorder - A pilot study. Br J Clin Psychol. 2017; 56(1): 1-15.

23. Kalleklev J, Karterud S. A comparative study of a mentalization- based versus a psychodynamic group therapy session. 2018. https://doi. org/10.1177/0533316417750987.

24. Byrne G, Egan J. A review of the effectiveness and mechanisms of change for three psychological interventions for borderline personality disorder. Clinical Social Work Journal. 2018; 46(3): 174-86.

25. Quek J, Melvin GA, Bennett C, Gordon MS, Saeedi $\mathrm{N}$, Newman LK. Mentalization in adolescents with borderline personality disorder: A comparison with healthy controls. J Pers Disord. 2019; 32(2): 145-63.

26. Hayden MC, Müllauer PK, Gaugeler R, Senft B, Andreas S. Improvements in mentalization predict improvements in interpersonal distress in patients with mental disorders. J Clin Psychol. 2018; 74(12): 2276-86.

27. Delavar A. Theoretical and practical research in the humanities and social sciences. $1^{\text {th }}$ ed. Tehran: Roshd Press. 2011.

28. First M, Spitzer R, Gibbon M, Williams JB. Structured clinical interview for DSM-IV Axis I disorders (clinician version) SCID-I administration booklet. Washington, DC: American Psychiatric Publishing. 1997.

29. Lobbestael J, Arntz A, Bernstein DP. Disentangling the relationship between different types of childhood maltreatment and personality disorders. J Pers Disord 2010; 24(3): 285-95.

30. Sharifi V, Asadi M, Mohammadi M, Amini H, Kaviani H, Semnani Y, et al. Reliability and feasibility of implementing the Persian version of diagnostic structured interview for DSM-IV (SCID). Advances in Cognitive Science. 2004; 6(1-2): 10-22.

31. Chegini M, Delavar A, Garrayi B. Psychometric characteristics of Millon clinical multiaxial inventoryIII. Journal of Psychology (Tabriz University). 2013; 8(29): $135-62$.

32. Sharifi AA, Molavi H, Namdari K. The validity of MCMI- III (Millon) scales. Knowledge \& Research in Applied Psychology. 2008; 34(9): 27-38.

33. Rahmani F, Kiani MA, Rezaie F, Nasuri M, Aras teh M. Personality, intellectual and emotional state of patients with borderline personality disorder. Scientific Journal of Kurdistan University of Medical Sciences. $2013 ; 18(3): 1-12$

34. Markstrom CA, Sabino VM, Turner BJ, Berman RC. The psychosocial inventory of ego strengths: Development and validation of a new Eriksonian measure. Journal of Youth and Adolescence. 1997; 26(6):705-32.

35. Altafi SH. Study and comparison of ego-strength and personality characteristics of substance-dependent and non-dependent individuals. (dissertation). Tehran: 
Shahed University; 2010.

36. Andrews G, Singh M, Bond M. The defense style questionnaire. Journal of Nervous and mental. 1993; 181(4): 246-56.

37. Besharat MA, Mohammadi Hoseini Nezhad E, Gholamali Lavasani M. The mediating role of cognitive emotion regulation strategies on the relationship between alexithymia, anger and anger rumination with ego defense styles. Contemporary Psychol. 2015; 9(2): 29-48.

38. Luyten P, Mayes LC, Fonagy P, Van Houdenhove B.
The interpersonal regulation of stress: A developmental framework. Manuscript submitted for publication. 2010.

39. Giugliano J. Object relations treatment of a woman with borderline personality disorder from a Mahlerian perspective: A case illustration. Clinical Case Studies. 2012; 11(6): 441-56.

40. Meulemeester CD, Lowyck B, Vermote R, Verhaest Y, Luyten P. Mentalizing and interpersonal problems in borderline personality disorder: The mediating role of identity diffusion. Psychiatry Res. 2017; 258: 141-4. 